\title{
Effect of garlic (Allium sativum) on lipid profiles, antioxidant activity and expression of scavenger receptor class B type I (SR-BI) in liver and intestine of hypercholesterolemic mice
}

\author{
Abbas Mohammadi ${ }^{1,2}$, Parham Norouzian $^{3}$, Mohammad Jamshidi ${ }^{4}$, Nader Najafi ${ }^{5}$, Ebrahim \\ Abbasi Oshaghi $^{4^{*}}$
}

${ }^{1}$ Department of Biochemistry, Afzalipour School of Medicine, Kerman University of Medical Sciences, Kerman, Iran

${ }^{2}$ Physiology Research Centre, Afzalipour School of Medicine, Kerman University of Medical Sciences, Kerman, Iran

${ }^{3}$ Department of Pharmacy, Shiraz University of Medical Sciences, International Branch, Shiraz, Iran

${ }^{4}$ Department of Biochemistry \& Nutrition, Medical School, Hamadan University of Medical Sciences, Hamadan, Iran

${ }^{5}$ Jiroft University of Medical Sciences, Jiroft, Iran

7abbasi@gmail.com (Corresponding author)

\begin{abstract}
Garlic is one of the medicinal plants, which has shown many useful effects, including Antioxidant activity, hypolipidemic and hypocholesterolemic effects, anti-hypertensive and anti-diabetic effects. Scavenger receptor class B type I (SR-BI) is a cell-surface receptor which mediated selective cholesterol ester uptake from the HDL particle. SR-BI also has an important role in cholesterol absorption in the intestine. Animals were randomly divided into three groups $(n=8)$; group 1: received chow + cholesterol $(2 \%)+$ cholic acid $(0.5 \%)$, group 2 : chow $+4 \%(w / w)$ garlic extract + cholesterol $(2 \%)+$ cholic acid $(0.5 \%)$, and group 3: chow only. After one-month mice were sacrificed, blood was collected; lipid profile and blood glucose were determined enzymatically as well as mRNA and protein levels of SR-BI were determined by RT-PCR and westernblot respectively. Compared with hypercholesterolemic control, garlic extract significantly decreased total cholesterol (TC), low-density lipoprotein cholesterol (LDL-C), triglycerides, Very Low-Density Lipoprotein-Cholesterol $($ VLDL-C) and atherogenic index (all of them $\mathrm{P}<0.05)$. The activity of Super oxide dismutase(SOD), glutathione $(G S H)$ and catalase levels were markedly increased in garlic-treated animals compared with hypercholesterolemic animals $(p<0.05)$. The plasma levels of MDA markedly reduced in garlic group compared with hypercholesterolemic group. Intestinal SR-BI mRNA and protein were significantly decreased in garlic extract mice treatment compared with hypercholesterolemic control. Levels of liver SR-BI protein significantly reduced in hypercholesterolemic group $(P<0.05)$. In conclusion, garlic extract markedly reduced TC, LDL-C, TG, VLDL-C and atherogenic index, as compared with the hypercholesterolemic control group. On the other hand garlic extract led to down-regulation of SR-BI protein and mRNA in the intestine of mice.
\end{abstract}

\section{Indexing terms/Keywords}

Cholesterol; Garlic; Intestine; Liver; SR-BI

Academic Discipline And Sub-Disciplines

Pharmaceutical Biochemistry

SUBJECT CLASSIFICATION

Anti Atherosclerosis

TYPE (METHOD/APPROACH)

Characterization, Biochemical and Pharmaceutical Study

\section{Council for Innovative Research}

Peer Review Research Publishing System

\section{Journal: Journal of Advances in Chemistry}

Vol. 5, No. 3

editor@cirworld.com

www.cirworld.com, member.cirworld.com 


\section{INTRODUCTION}

High levels of total cholesterol and low-density lipoprotein cholesterol (LDL-C) along with low serum levels of high-density lipoprotein cholesterol (HDL-C) is contributing to the development of atherosclerosis [1]. In this respect, garlic extracted (Allium sativum) is a food component which usually used in our food. Furthermore, garlic extract have been shown to be applicable in atherosclerosis, because this herb has many useful properties, including; Antioxidant activity, antihypertensive activity, hypolipidemic and hypocholesterolemic effects, anti-diabetic, anti-hyperhomocysteinemia and antithrombotic effects [2]. Scavenger receptor class B member 1 (SR-BI) is a cell-surface receptor which mediated selective cholesterol ester uptake from the center of the HDL particle. In this process cholesterol, ester is transferred to the liver cell without degradation of this protein [3]. SR-BI is expressed with high levels in the liver and steroidogenic cells. Mutations in the SR-BI gene in mice who is inhibited or reduced SR-BI expression leads to rise in plasma HDL-C concentrations and decrease HDL-CE uptake by the liver [4]. On the other hand, transgenic mice expression of high levels of liver SR-BI leads to decrease in serum levels of high-density lipoprotein (HDL) cholesteryl ester (CE), Apolipoprotein AI (Apo AI), and Apolipoprotein All (Apo All) [5].

The molecular mechanisms of cholesterol absorption in the intestine are yet not entirely understood. However, many proteins, including scavenger receptor BI (SR-BI), Niemann-Pick C1-like 1 (NPC1L1), aminopeptidase N and ATPbinding cassette transporter G5/G8 (ABCG5/G8) have been proposed as candidates of cholesterol transporters or receptors [6].The present study was performed to investigate the effect of garlic extract on altered biochemical parameters and expression of SR-BI in liver and intestine of N-marry mice.

\section{EXPERIMENTAL PROCEDURE}

Male N-Mary mice weighing 28-30 g each were kept in animal cages, maintained on $12 \mathrm{~h}$ light/12 $\mathrm{h}$ dark cycle cycles at room temperature of $22 \pm 1^{\circ} \mathrm{C}$. They were fed commercial store diet and water. Animals acclimatized for one week and then were randomly divided into three groups $(n=8)$ : group 1 received chow + cholesterol $(2 \%)+$ cholic acid $(0.5 \%)$, group 2 : chow $+4 \%(w / w)$ garlic extract + cholesterol $(2 \%)+$ cholic acid $(0.5 \%)$, and group 3: chow only. Triglyceride, cholesterol and blood glucose, were at the baseline prior to treatment. Animals were supervised every day, and body weight was recorded every $48 \mathrm{hrs}$. After one-month treatment, the mice were anesthetised with sodium thiopental and sacrified and then blood samples were collected from heart. After that, blood samples allowed to clot, and was centrifuged at $3000 \mathrm{~g}$ for 10 minutes and then stored at $-20^{\circ} \mathrm{C}$ until assay. Liver and intestine were removed, washed with Phosphate buffered saline (PBS) and stored in $-70{ }^{\circ} \mathrm{C}$ until use. All experiments procedure of this study was approved by the Animal Research Ethic Committee of Kerman Medical University (Kerman, Iran). Fasting blood glucose, triglyceride, total cholesterol and HDL-C were measured enzymatically. The LDL-C and VLDL-C were calculated using Friedwald equation. Atherogenic index was calculated by using the following formula [7-9]:

$$
\text { Atherogenic index }=\frac{\text { total cholesterol }-(\mathrm{HDL}-\mathrm{C})}{\mathrm{HDL}-\mathrm{C}}
$$

\section{Antioxidant enzyme assays and Lipid peroxidation}

The superoxide dismutase (SOD) activity (Misra and Fridovich method), Catalase (CAT) (Aebi method) was determined, Reduced glutathione (GSH) (Beutler E, method) and MDA levels (Ohkawa method) were determined [9].

\section{Garlic extract}

The garlic cloves were purchased from market of medicinal herbal in Kerman city, and then were washed with distilled water, and dried. Twenty grams of shadow dried powder dissolved in $200 \mathrm{ml}$ of distilled water at room temperature for 24 h. the extract filtrated and after that was dried at $40^{\circ} \mathrm{C}$ in an incubator. The extract was kept in dark vials at $-20^{\circ} \mathrm{C}[10]$.

\section{Reverse transcriptase PCR}

Total RNA from liver and intestine were extracted using Accuzol Reagent and also cDNA synthesis was performed according to the manufacturer's instructions (Bioneer, Korea and Fermentas, Lithuania respectively). For reverse transcriptase PCR (RT-PCR) reaction, 35 cycles of PCR amplification were achieved with denaturation at $95 \stackrel{\circ}{\circ}$ for $30 \mathrm{~s}$, annealing at $63^{\circ} \mathrm{C}$ for $30 \mathrm{~s}$, and extension at $72{ }^{\circ} \mathrm{C}$ for $30 \mathrm{~s}$ with using PCR machine. Finally, all PCR reactions were completed with a single extension cycle at $72{ }^{\circ} \mathrm{C}$ for 5 minutes. The products were electrophoresed on a $2.5 \%$ agarose gel and visualized by staining with ethidium bromide. The following primers were used in this study. Mouse Beta actin Primer; forward (F): 5'-TGG AAT CCT GTG GCA TCC ATG AAA C-3' and reverse (R): 5'-TAA AAC GCA GCT CAG TAA CAG TCC G-3'. Mouse SR-BI primer; forward (F): 5'-CAC CTT CAA TGA CAA CGA CAC C-3'and reverse (R): 5'-TCT CTG AGC CAT GCG ACT TG-3' [8].

\section{Western blot analysis}

Fifty milligram of liver or intestine were homogenized in $700 \mu \mathrm{l}$ of RIPA buffer containing $1 \mu \mathrm{M}$ PMSF and $1 \%$ protein inhibitor cocktail (Santa Cruz, USA), after that centrifuged (14000 rpm) at $4{ }^{\circ} \mathrm{C}$ for 15 minutes. The extracted total proteins $(60 \mu \mathrm{g})$, were separated onto a $12.5 \%$ SDS-PAGE gel and were transferred onto a PVDF membrane (Roche Applied Science). The membrane was incubated with SR-BI (1:2500 dilution, Novus Biological) for $1.5 \mathrm{~h}$ at room temperature after blocking with $2 \%$ skim milk in Tris-buffered saline containing Tween-20 (TBS-T) (Roche Applied Science) for $2 \mathrm{hrs.}$ The blots then were incubated with horseradish peroxidase-conjugated anti-rabbit IgG (1:10 000 dilution, Roche Applied Science) at room temperature for $1.5 \mathrm{~h}$. ECL Western blot analysis system (Roche Applied Science) was used to detect bound antibodies. The same membranes were reprobed with anti- $\beta$-actin antibodie (1:2500 dilution, Novus Biological) to 
verify the equal loading of proteins prepared from liver and intestine after different treatments. Density of bands was determined with Lab Work analyzing software (UVP, UK), and data are expressed as the percent ratio of the SR-BI to $\beta-$ Actin $[11,12]$.

\section{Statistical analysis}

The differences between treatment groups were analyzed by ANOVA (Tukey). All results are presented as mean \pm S.E.M and differences with at $\mathrm{P}<0.05$ were considered significant.

\section{Results}

\section{Body weight and Biochemical analysis}

Table 1 shows body weight, FBS, TC, LDL-C, HDL-C, TG and VLDL-C, in mice fed a high-cholesterol diet, garlic extract and chow. Body weight show significant increase in hypercholesterolemic control compared with chow diet; while garlic non-significantly reduced body weight. Reduction of Fasting Blood Sugar (FBS) in garlic-treated animals was not significant compared with hypercholesterolemic group. Serum TC, LDL-C, TG, VLDL-C and atherogenic index significantly decreased in garlic extract in comparison with hypercholesterolemic mouse. Change in Serum HDL-C between garlic extract and hypercholestrolemic groups were not significant in comparison with chow.

\section{Antioxidant activity}

The activity of SOD was markedly increased in garlic group $(p<0.05)$ when compared with hypercholesterolemic group. The levels GSH and catalase were also markedly increased in garlic group $(p<0.05)$ compared with hypercholesterolemic animals. The plasma concentration of MDA significantly reduced in garlic group compared with hypercholesterolemic animals $(\mathrm{p}<0.05)$ (table 2).

\section{Levels of SR-BI protein and mRNA}

Immunoblot analysis of the intestine and liver protein probed with anti SR-BI revealed bands with expected size of $82 \mathrm{KDa}$. Intestinal SR-BI protein significantly reduced in garlic extract $(P<0.05)$. Liver $S R-B I$ significantly reduced in response to the hypercholestrolemic diet (combination of $2 \%$ cholesterol and $0.5 \%$ cholate) (hypercholesterolemic group) $(P<0.05)$, while the change of SR- BI in liver of garlic extract group was not significant (Figure 1). PCR products of SR-BI showed an expected band of $82 \mathrm{bp}$. Intestinal SR-BI mRNA significantly reduced in garlic extract $(\mathrm{P}<0.05)$. Liver SR-BI mRNA had not been a significant change between all groups (Figure 2).

Table 1: Comparison of biochemical factors between different treatment groups

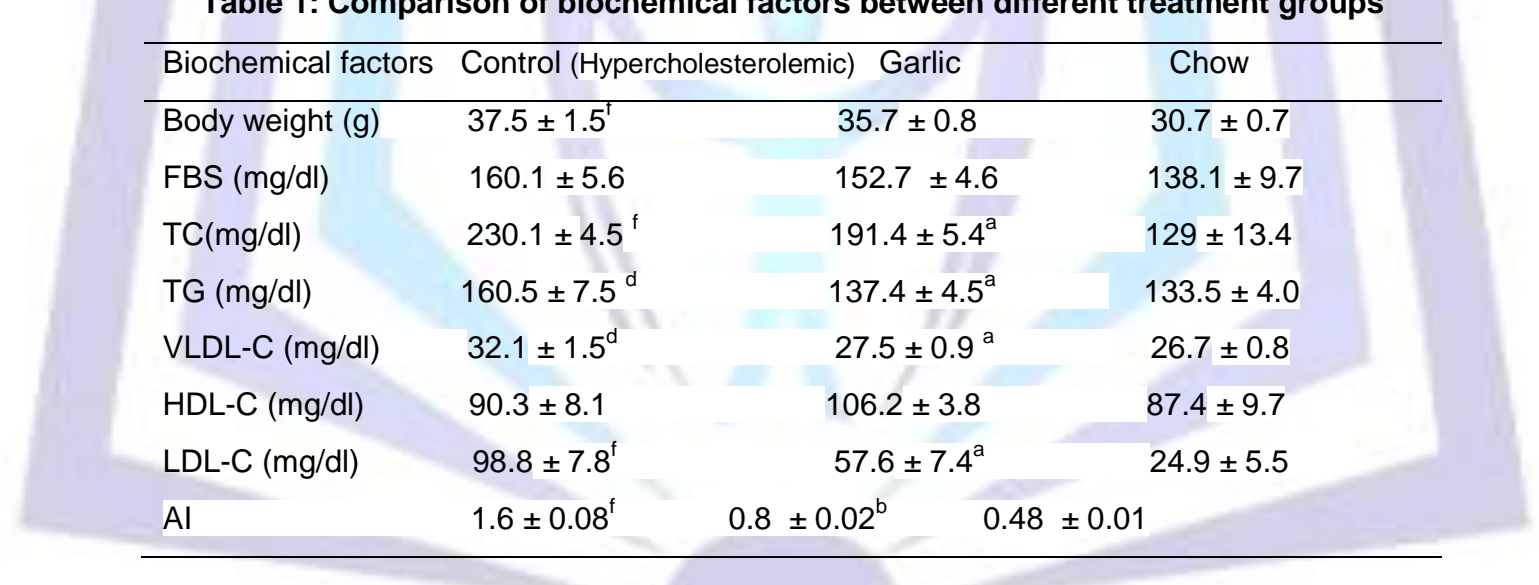

FBS: Fasting blood sugar, TC: Total cholesterol, TG: Triglyceride, HDL-c: High-density lipoprotein cholesterol, LDL-c: Low-density lipoprotein cholesterol, VLDL-c: Very low-density lipoprotein cholesterol, Al: atherogenic index. ${ }^{a} p<0.05$ considered as significant compared with Hypercholestrolemic. ${ }^{b} p<0.01$ compared with Hypercholestrolemic. ${ }^{c} p<0.001$ compared with Hypercholesterolemic. ${ }^{d} p<0.01$ compared with chow. ${ }^{f} p<0.001$ compared with chow. Data represent as mean \pm SEM $(n=8)$.

Table 2: Antioxidant enzyme activities in the plasma of animals

\begin{tabular}{|c|c|c|c|c|}
\hline & SOD(units/ml) & MDA (nmol/l) & $\mathrm{GSH}(\mu \mathrm{mol} / \mathrm{ml})$ & Catalase(units/ml) \\
\hline Chow & $112.5 \pm 6.0$ & $2.3 \pm 0.16$ & $6.33 \pm 0.66$ & $63.3 \pm 5.9$ \\
\hline garlic & $120.0 \pm 6.1^{a}$ & $2.5 \pm 0.18^{a}$ & $6.08 \pm 0.45^{a}$ & $60.1 \pm 5.1^{\mathrm{a}}$ \\
\hline Hypercholesterolemic & $141.1 \pm 7.3$ & $3.9 \pm 0.20$ & $4.17 \pm 0.30$ & $45.3 \pm 6.4$ \\
\hline
\end{tabular}

Data are expressed as mean \pm SEM. ${ }^{a} p<0.05$ compared to Control (Hypercholesterolemic). SOD: Super oxide dismutase, MDA: malondialdehyde, GSH; glutathione. 

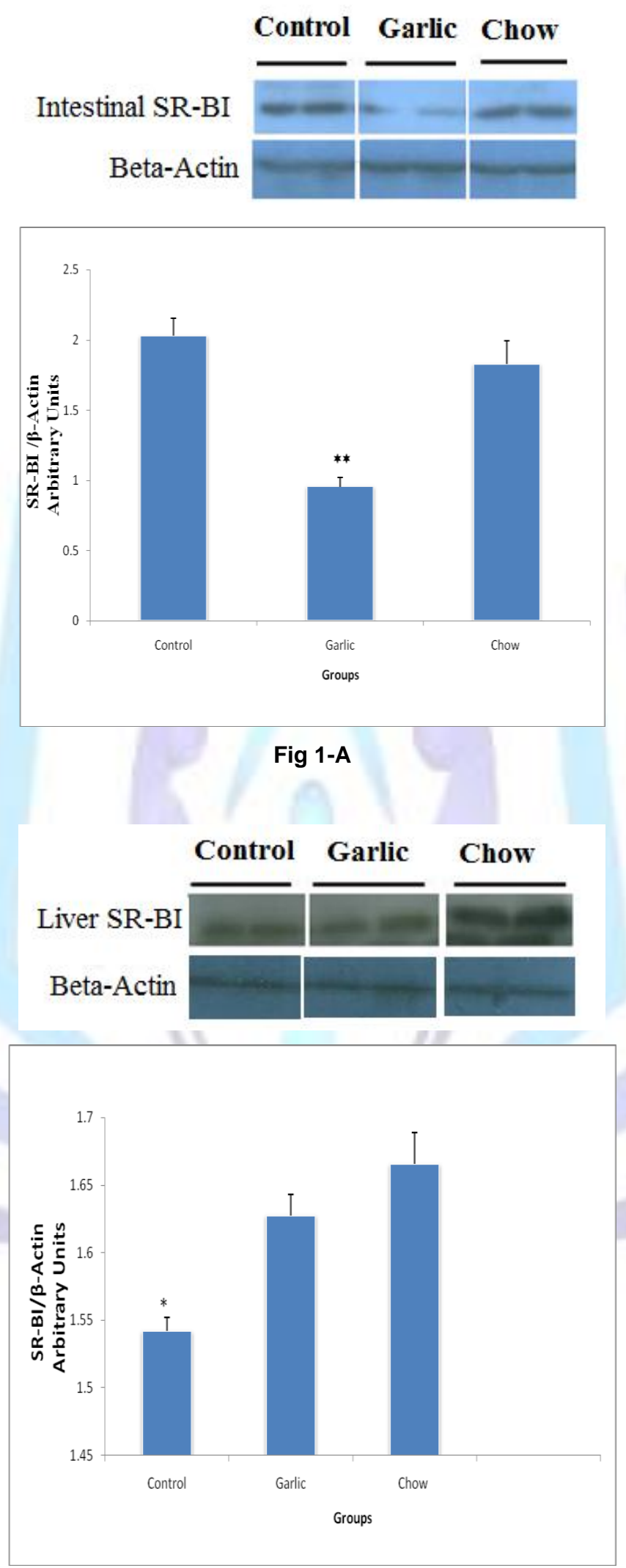

Fig 1-B

Fig 1: Westernblot of SR-BI protein in intestine and liver of animals which treated differently. Fig 1-A: The average band density ratio of intestinal SR-BI proteins in three groups including $(n=8)$; control (hypercholesterolemic), garlic and chow received. Data in the graph are represented as mean $\pm S E M$. ${ }^{* *} \mathrm{P}<0.01$ versus chow animals. Fig1-B: The average band density ratio of liver SR-BI proteins in three groups including $(n=8)$; control (hypercholesterolemic), 
garlic and chow received. Data in the graph are represented as mean \pm SEM. $\beta$-Actin was used as an internal control. The density of liverl SR-BI protein significantly reduced in the in the high cholesterol diet (control) group compared with the chow. ${ }^{*} P<0.05$ versus chow animals.

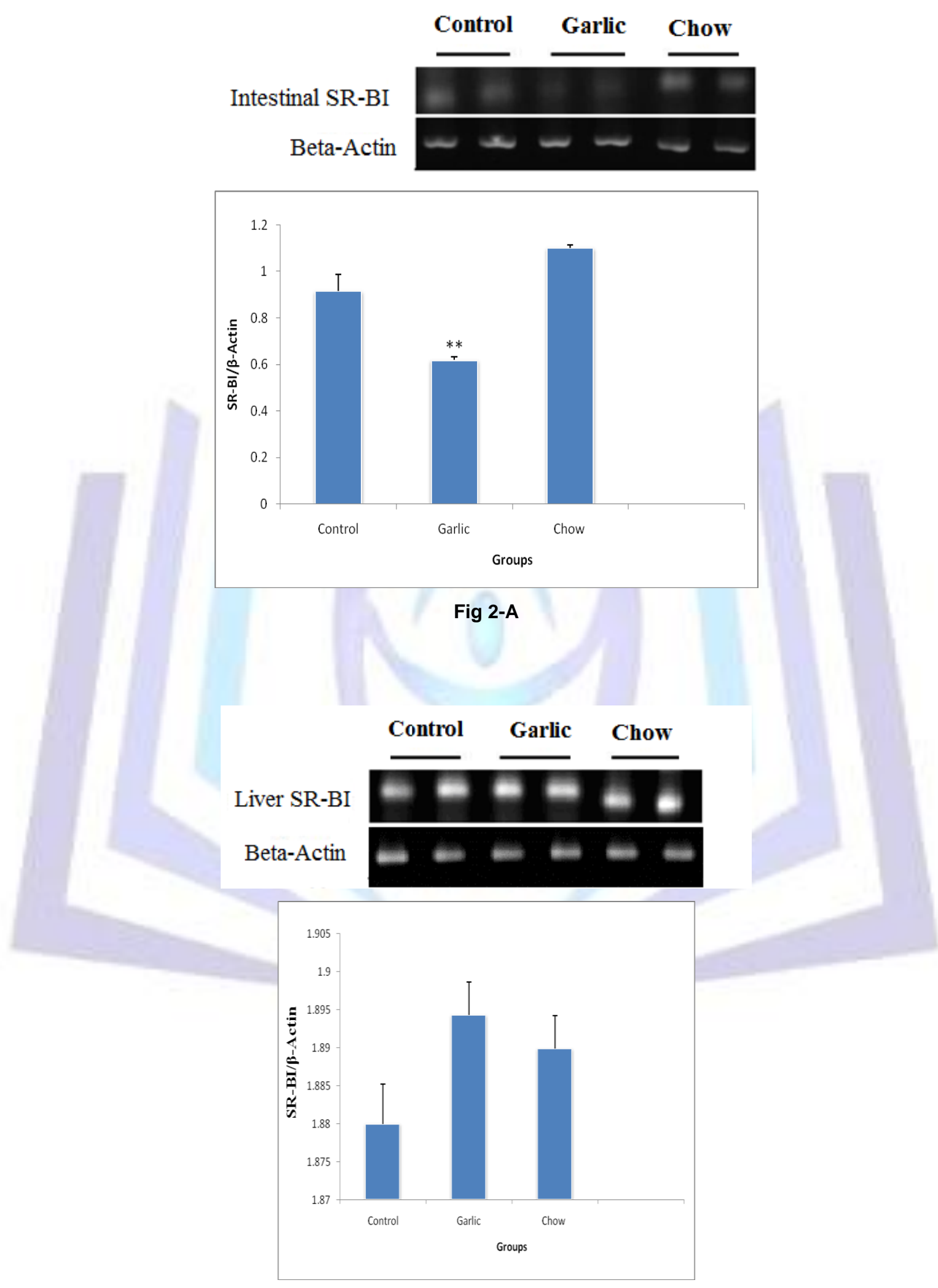

Fig 2-B

Figure 2: Reverse Transcription PCR of SR-BI protein in intestine and liver of animals which treated differently. Fig 2-A: The average band density ratio of intestinal SR-BI mRNA in three groups including ( $n=8)$; control (hypercholesterolemic), garlic and chow received. Data in the graph are represented as mean \pm SEM. The density of 
intestinal SR-BI mRNA significantly reduced in the in the garlic group compared with the chow. ${ }^{* *} \mathrm{P}<0.01$ versus chow animals. Fig 2-B: The average band density ratio of liver R-BI mRNA in three groups including ( $n=8)$; control (hypercholesterolemic), garlic and chow received. Data in the graph are represented as mean \pm SEM. $\beta$-Actin was used as an internal control. The change of liver SR-BI mRNA was not significant in all treatment groups.

\section{Discussion}

Many experiments using rabbit and rat have shown that garlic consumption significantly decreased the content of total serum cholesterol, LDL, VLDL, and also increased the level of HDL [2]. Effendy, Simmons, Campbell, et al. results show that treatment with aged garlic extract (AGE) in hypercholesterolemic rabbits significantly reduced accumulation of cholesterol in vessel wall and also inhibited development of arteriosclerotic plaques in the arterial wall [2]. In humans and animals study allicin, and its derivative are known as the major active compound responsible for the hypocholesterolemic and hypolipidemic effects of garlic.

In our experiment, fed with high cholesterol diets significantly increased serum triglycerides, total cholesterol, LDL-C and VLDL-C.

Our result was agreement with Kamanna VS, et al [13]. experiment which showed that, the increased levels of LDL-C and cholesterol in animals which fed the hypercholestrolemic diet were partly reversed in mice receiving a supplement of garlic powder.

In this study supplementation of diet with $4 \%$ of garlic extract caused a significant reduction in the serum total cholesterol (16\%), triglycerides (14.4\%), LDL-C (41\%),VLDL-C (15\%) and atherogenic index (50\%) in mice. Previous studies have shown that treatment with garlic inhibited hepatic fatty acid synthesis and as a result reduced the accumulation of lipid in the liver, and the triglycerides level in the plasma [14]. In the present study there was non-significant reduction in blood glucose in garlic extrac. The prior reports $[15,16]$ showed that garlic had not influence on the concentration of serum glucose.

A diversity of internal antioxidants including superoxide dismutase, catalase, glutathione peroxidase and peroxidase are present in organisms which protect them from oxidative stress. Antioxidants play vital role in maintenance of wellbeing and regular cellular activity. They protect body cells by stabilizing and deactivating of free radicals [9]. Moreover, peroxidation of lipid is one of the early events happening during in vitro oxidation of LDL, and consequently atherosclerosis formation. In the vessel wall atherogenic lipids especially oxidized low-density lipoprotein (ox-LDL) are responsible for a wide range of cellular dysfunctions [9]. The high MDA levels are recognized as a positive sign of lipid peroxidation. There was a significant raise in SOD, GSH and catalase in garlic-treated group. The MDA levels markedly reduced in in garlic-treated group compared with hypercholesterolemic group.

SR-BI is one of the lipid transporters which have important role in lipid metabolism. SR-BI is an $82 \mathrm{kDa}$ membrane protein highly expressed in liver, intestine and steroidogenic tissues. In the liver, SR-BI is the chief receptor which mediates selective uptake of cholesterol ester from plasma HDL [17].

The results of our study have showed that liver SR-BI protein significantly down-regulated in an atherogenic diet (cholesterol $2 \%$ and cholic acid $0.5 \%$ ). This finding is similar to Niemeier A, et al. [18] and Hong S, et al. [19] results that showed an atherogenic diet significantly reduced posttranslational expression of hepatocyte SR-BI protein levels. This reduction is maybe atherogenic diet leads to rise of liver cholesterol. We observed a non-significant reduction in HDL-C levels in hypercholesterolemic mice. In this study change of SR-BI protein and mRNA in garlic extract group was not significant.

Absorption of lipid in intestine is an important contributor to the general balance of lipid metabolism. Diverse proteins have consecutively been concerned in intestinal lipid absorption. For example, CD-36 a multiligand scavenger receptor has projected to take up fatty acids and monoglycerides, however, could also contribute in cholesterol absorption. The other transporter is a Niemann-Pick C1 Like 1 (NPC1L1), which has an important role in cholesterol absorption. Intestinal cholesterol absorption in NPC1L1 knock-out mice decreased by $80 \%$. In addition, aminopeptidase-N has been implicated in cholesterol absorption [3]. SR-BI is a main intestinal transport protein which inhibited by a potent cholesterol absorption inhibitor, SCH354909, derivative of ezetimibe [20]. SR-BI is expressed in membranes of enterocyte brush border, mostly at the apex side of intestinal villosities and also in the proximal piece of the intestine where cholesterol is mainly absorbed [3].

The gastrointestinal tract is known as a main potential therapeutic target in dyslipidemia management. Restriction of dietary cholesterol and triglyceride has been utilized as the primary therapy in the whole-body cholesterol regulation [21]. $\mathrm{SR}-\mathrm{BI}$ is one of the important intestinal cholesterol absorption transporters. In this experiment, SR-BI was significantly reduced in an intestine by garlic extract in comparison with control. Hauser $\mathrm{H}$ et al. reported that SR-BI played major role in absorption of cholesterol [22]. The ability of ezetimibe to inhibit of SR-BI proposed that this protein has an important role in cholesterol absorption. Altmann SW et al. also reported that SR-BI inhibited by SCH354909 (derived from ezetimibe) and has a vital role in intestinal cholesterol absorption (20). 


\section{Conclusion}

Interestingly, our results suggested that the garlic extract significantly decreased the TC, LDL-C, TG, VLDL-C and atherogenic index, as compared with the control group. Taken together, we confirmed that garlic extract dramatically suppress the atherosclerosis risk by improve antioxidant activity and also by reducing total cholesterol and triglyceride absorption via inhibition of intestinal SR-BI. Thus, we conclude that the garlic extract have beneficial effects in lowering the incidence of cardiovascular disease (CVD) and atherosclerosis by reducing the cholesterol and triglyceride absorption.

\section{Acknowledgments}

This study was funded by Kerman University of Medical Sciences. We would also like to thank Professor Khosrow Adeli from Hospital for Sick Children, Toronto, Canada, for his advice.

\section{References}

[1] Johnson, M.S., Svensson, P.A., and Helou, K.1998. Characterization and Chromosomal Localization of Rat Scavenger Receptor Class B Type I a High Density Lipoprotein Receptor with a Putative Leucine Zipper Domain and Peroxisomal Targeting Sequence. Endocrinology. 139:72-80.

[2] Corzo-Martinez, M., Corzo, N., and Villamiel, M. 2007. Biological properties of onions and garlic. Trends in Food Science \& Technology. 18: 609-625.

[3] Rhainds, D and Brissette, L. 2004 .The role of scavenger receptor class B type I (SR-BI) in lipid trafficking Defining the rules for lipid traders. Int. J. Biochemistry \& Cell Biology. 36: 39-77.

[4] Varban, M.L., Rinninger, F., and Wang, N. 1998. Targeted mutation reveals a central role for SR-BI in hepatic selective uptake of high density lipoprotein cholesterol. Proc. Natl. Acad. Sci. USA. 95: 4619-4624.

[5] Wang, N., Arai, T., and Ji, Y. 1998. Liver specific overexpression of scavenger receptor BI decreases levels of very low density lipoprotein apoB, low density lipoprotein apo $B$, and high density lipoprotein in transgenic mice. J. Biol. Chem. 273: 32920-32926.

[6] Kramer, W., Girbig, F., and Corsiero, D. 2005. Aminopeptidase N (CD13) is a molecular target of the cholesterol absorption inhibitor ezetimibe in the enterocyte brush border. J. Biological Chemistry. 280 (2), 1306-1320.

[7] Mohammadi, A., Mirzaei, F., and Abbasi Oshaghi. E. 2013. Effect of flaxseed on Serum Lipid Profile and expression of NPC1L1, ABCG5 and ABCG8 genes in the intestine of diabetic rat. Avi $J$ Med Biochem. 1(1): 1-6.

[8] Mohammadi, A., E. Abbasi Oshaghi, and Noori Sorkhani, A. 2012. Effect of Opium on Lipid Profile and Expression of Liver X Receptor Alpha (LXRa) in Normolipidemic Mouse. FNS. 3(2): 249-254.

[9] Mohammadi, A., Mirzaei, F., Abbasi Oshaghi. E., and et al. 2013. The in vivo biochemical and oxidative changes by ethanol and opium consumption in Syrian hamsters. IJB. 5:14-23.

[10] Mohammadi, A., and Abbasi Oshaghi, E, Effect of garlic on lipid profile and expression of LXR alpha in intestine and liver of hypercholesterolemic mice. Journal of Diabetes \& Metabolic Disorders. 2013: in press.

[11] Abbasi Oshaghi. E., A. Noori Sorkhani, A. Rezaei, 2012. Effects of Walnut on Lipid Profile as Well as the Expression of Sterol-Regulatory Element Binding Protein-1c (SREBP-1c) and Peroxisome Proliferator Activated Receptors a (PPAR $\alpha$ ) in Diabetic Rat. FNS. 3(2): 255-259.

[12] Mohammadi, A., Mirzaei, F., Abbasi Oshaghi. E., and et al. 2013. Influence of flaxseed on lipid profiles and expression of LXR $\alpha$, in intestine of diabetic rat. IJB. 5:23-28.

[13] Kamanna, V.S., and Chandraseklara, N.1982. Effect of garlic (Allium sativum linn) on serum lipoproteins and lipoprotein cholesterol levels in albino rats rendered hypercholesteremic by feeding cholesterol. Lipids., 17: 4838.

[14] Kritchevsky, D., S.A. Tepper, R. Morrisey and Klurfeld, D. 1980. Influence of garlic oil on cholesterol metabolism in rats. Nutr Rep Int. $22: 641-5$.

[15] Ali, M., and Thomson, M. 1993. Consumption of garlic clove a day could be beneficial in preventing thrombosis. PLEFA. 53:211-212.

[16] Jain, A.K., Vargas, R. S., and Gotzkowsky, F.G.1993. Can garlic reduce levels of serum lipids? A controlled clinical study. Am. J. Med. 94:632-635.

[17] Wiersma, H., Gatti, A., and Nijstad, N.1998. Scavenger Receptor Class B Type I Mediates Biliary Cholesterol Secretion Independent of ATP-Binding Cassette Transporter g5/g8 in Mice. Hepatology. 50:1-10.

[18] Niemeier, A., Kovacs, W.J., and Strobl, W. 2009. Atherogenic diet leads to posttranslational down-regulation of murine hepatocyte SR-BI expression. Atherosclerosis. 202:169-175. 
[19] Hong, S.C., Zhao, S.P., and Wu, Z.H. 2007. Effect of probucol on HDL metabolism and class B type I scavenger receptor (SR-BI) expression in the liver of hypercholesterolemic rabbits. Int. J. Cardio. 115(1): 29-35.

[20] Altmann, S.W., Davis, H.R., and Yao, X. 2002. The identification of intestinal scavenger receptor class B, type I (SR-BI) by expression cloning and its role in cholesterol absorption. J. BBA. 1580: 77-93.

[21] Manhas, A., and Farmer, J.A. 2004. Hypolipidemic therapy and cholesterol absorption. J. CHD. 6:89- 93.

[22] Hauser, H., Dyer, J.H., and Nandy, A.1998. Identification of a receptor mediating absorption of dietary cholesterol in the intestine. Biochemistry. 37:17843-17850. 